\title{
miR-145 is associated with placental growth in mice
}

\section{Lisa Jones, Jayne Charnock and Karen Forbes}

Maternal \& Fetal Health Research Centre, University of Manchester, St Mary's Hospital, UK

\section{Introduction}

- Abnormal placental development and function can result in fetal growth restriction (FGR).

- FGR is associated with increased risk of infant morbidity and mortality and has life-long impacts on health.

- The insulin-like growth factor (IGF) axis is imperative for normal placental and hence fetal development .

- The actions of IGF-I and -II in regulating both human and murine placental development and function are

mediated by IGF1R (1,

- Modulating expression of IGF1R in the placenta may improve placental growth and improve outcomes of pregnancies complicated by FGR

- In the human placenta, microRNAs (miRs) regulate expression of components of the IGF axis $(3,4)$.

- One miR- miR-145- modulates human placental growth by targeting IGF1R ${ }^{(4)}$, so it may be possible to utilise miR-145 based drugs to manipulate placenta growth in humans.

- Prior to testing in humans, miR-based drugs would need to be tested in mice, however it is unclear whether miRs and specifically miR-145, influence placental and fetal growth in mice.

\section{Hypotheses and Aims}

\section{Hypotheses:}

$>$ miR-145 is expressed in the mouse placenta

miR-145 regulates murine placental and fetal growth by targeting IGF1R

Aims:

- To ascertain whether miR-145 is expressed in the mouse placenta and fetus

- To determine if miR-145 is associated with placental growth in mice by examining both placenta growth and miR-145 expression across gestation

- To determine the relationship between miR-145 expression and IGF1R in the mouse placenta and in the fetus

\section{Methods}

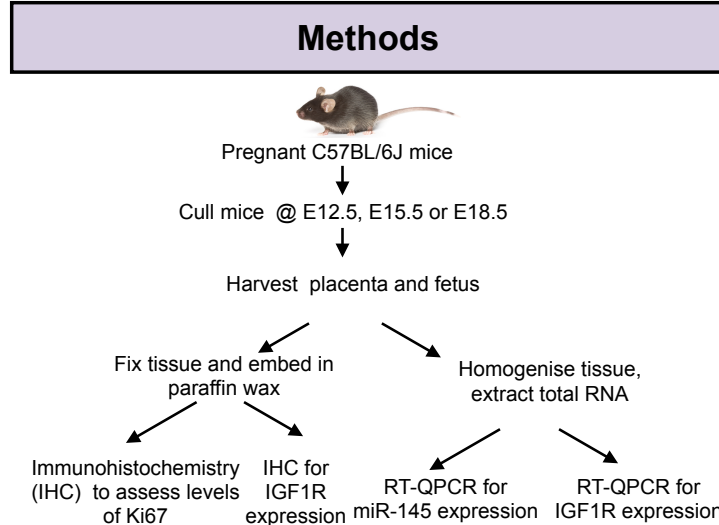

of Ki67 expression miR-145 expression IGF1R expression

- miR-145 and IGF1R were quantified by RT-QPCR using specific primers and values were normalised to $5 s$ and $18 \mathrm{~s}$ rRNA, respectively.

- IGF1R expression was assessed using IHC.

- Levels of proliferation were analysed by performing IHC for Ki67 and expressing the number of Ki67+ cells as a percentage of total number of nuclei (haematoxylin stained).

\section{Results}

1) miR-145 is expressed in murine placenta and inversely correlates with placental growth

miR-145 Expression - Fetus

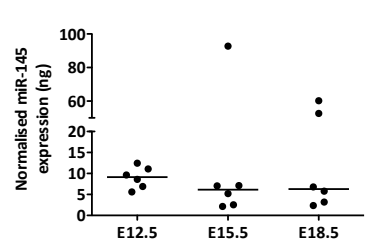

miR-145 Expression - Placenta

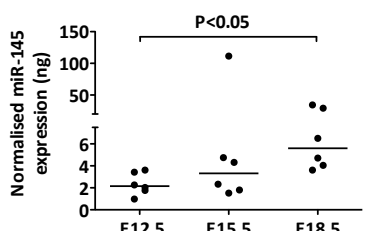

Placental Proliferation (Ki67)

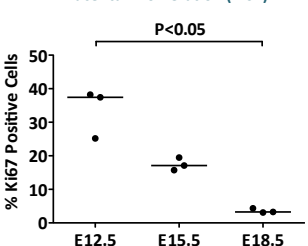

- $\mathrm{QPCR}$ demonstrated that miR-145 is expressed in the mouse fetus but does not alter with gestation

- QPCR confirms that miR-145 is expressed in the mouse placenta, and levels significantly increase $(P<0.05)$ over gestation.

- Analysis of cell proliferation (Ki67) reveals that proliferation significantly decreases $(P<0.05)$ over gestation.

- These results are consistent with results found in studies on the human placenta ${ }^{(4)}$ and suggest that miR-145 may negatively regulate placental growth in mice.

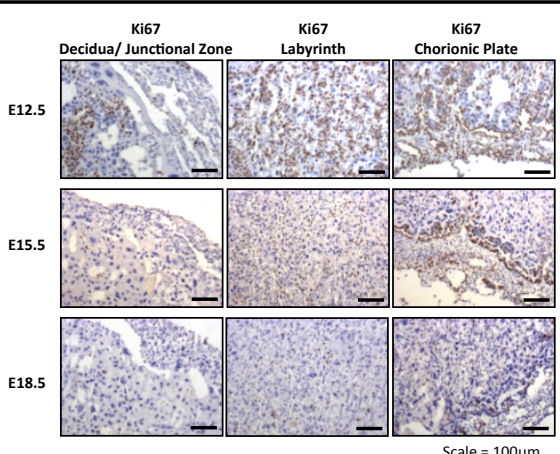

\section{2) IGF1R is expressed in the fetus and placenta throughout gestation}

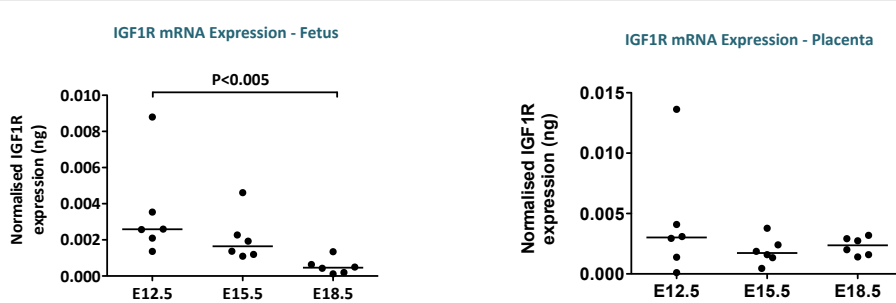

- IHC demonstrates that IGF1R is expressed throughout the placenta (decidua, junctional and labyrinth zones) in mice and protein expression appears to reduce with gestation.

protein expression appears to reduce with gestation.
- However, QPCR confirms that IGF1R mRNA expression does not alter $(P>0.05)$ in the mouse placenta over gestation.

- These results are consistent with the human placenta whereby studies have shown that miR-145 acts by reducing IGF1R

protein and not mRNA expression (3).

- QPCR also demonstrated that IG1R mRNA is expressed in the mouse fetus and this significantly decreases $(P<0.005)$ over pregnancy.

\section{Conclusions}

- miR-145 is expressed in the mouse placenta and is significantly increased over gestation, similar to data observed in the human placenta ${ }^{(4)}$

- Proliferation significantly decreases over gestation in the mouse placenta, and inversely correlates with placental growth; this is also consistent with studies in the human placenta.

- There was no change in placental IGF1R mRNA throughout pregnancy, however IHC appears to demonstrated that IGF1R protein expression decreases towards term; this discrepancy between mRNA and protein expression is consistent with the known actions of miRs in the human placenta: data from this lab group suggests that miR-145 targets IGF1R protein degradation as opposed to repression of mRNA translation (4)

- These results combined suggests that miR-145 is likely to have similar actions in regulating placental growth in mice as it does in humans, however the role of miR-145 in regulating IGF1R expression in mice remains to be established.

- Ongoing work will examine the potential therapeutic role of miR-145 based drugs to improve placental and fetal growth using mouse models of FGR.

\section{References}

Forbes K, Westwood M, Baker PN and Aplin JD (2008) Insulin-like growth factor I and II regulate the life cycle of trophoblast in the developing human placenta. Am J Physiol Cell Physiol, 294 (6):C1313-22
Roberts CT, Owens JA, Sferruzzi-Perri AN. (2008) Distinct actions of insulin-like growth factors (IGFs on placental development and fetal growth: lessons from miceand guinea pigs. Placenta Mar; 29 Suppl A:S42-7.

Farrokhnia F, Westwood M, Aplin JD and Forbes K (2012) Identification of microRNAs involved in regulating growth factor signalling in the first trimester placenta. $6^{\text {th }}$ International Conference of the GRS and IGF Society, Munich, Germany. Growth Hormone 